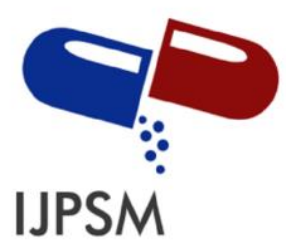

Dr. C.Pandian et al, International Journal of Pharmaceutical Sciences and Medicine (IJPSM),

Vol.6 Issue. 12, December- 2021, pg. 1-17

ISSN: 2519-9889

Impact Factor: 5.365

\title{
FORMULATION DEVELOPMENT AND CHARACTERIZATION OF TRIAMCINOLONE LOADED CUBOSOMES FOR TRANSDERMAL DRUG DELIVERY
}

\author{
"Dr. C.Pandian; Dr. A.Abdul Hasan Sathali; G. Abirami; E. Krithika \\ Department of Pharmaceutics, College of Pharmacy, Madurai Medical College, Madurai-20 \\ Affiliated to the Tamilnadu Dr. M.G.R. Medical University, Chennai-32, Tamilnadu \\ *Corresponding author e-mail id: drcpandian73@gmail.com
}

DOI: 10.47760/ijpsm.2021.v06i12.001

\begin{abstract}
Psoriasis is a chronic condition that is caused by the negative signals given by immune system, which leads to hyperproliferation and other inflammatory reactions on the skin. These conditions may adversely affect the quality of the patient's life leading to psychological stress. Topical delivery of drug is always preferred for Psoriasis because other treatments may lead to systemic intoxication and other adverse reactions. Triamcinolone is a topical corticosteroid belonging to BCS class IV (low solubility and permeability) used to treat Psoriasis. The limitations with transdermal delivery is that only a small amount of the drug can be transferred through the skin tissue due to the barrier effects of the Stratum corneum. Therefore, Novel transdermal delivery system, Cubosomes belonging to Nanostructured lipid carriers were chosen to overcome the issues of solubility and permeability. Twelve formulations were prepared with various ratios of Glyceryl monooleate (2.5 to 5\%) \& Poloxamer 407 (0.5 to $2 \%$ ) and the formulations were evaluated for particle size, PDI, zeta potential, entrapment efficacy, drug content and in-vitro release. The best composition of Cubosomes was selected and incorporated into transdermal patch and the formulated patches were evaluated.
\end{abstract}

KEYWORDS: Cubosomes, Transdermal patch, Permeability

\section{INTRODUCTION}

Psoriasis is a skin disorder that is chronically proliferative and inflammatory. The extensor surfaces, scalp, and lumbosacral area are all affected, with erythematous plaques coated with silvery scales. The pathogenesis involves activated $\mathrm{T}$ lymphocytes infiltrating the skin and stimulating keratinocyte production. The formation of thick plaques is caused by a disruption in keratinocyte turnover. Other symptoms include epidermal cells failing to release lipids, resulting in flaky, scaly skin, which is typical of Psoriasis. [1] It is linked to a number of serious medical issues, including depression, psoriatic arthritis, and cardiometabolic syndrome. Although psoriasis cannot be cured, it can be managed to reduce physical and psychological harm by treating patients early in the disease process, recognising and preventing related multimorbidity, establishing lifestyle changes, and using a personalised therapy approach. [2] The scalp, elbows, knees, umbilicus, genitalia, sacrum, and shins are the most typically affected areas. Emollients, dithranol, tar, and corticosteroids are the first-line therapies. Phototherapy and systemic medicines are examples of second-line treatments that have more adverse reactions. [3] 


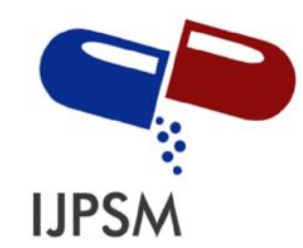

\section{Dr. C.Pandian et al, International Journal of Pharmaceutical Sciences and Medicine (IJPSM), Vol.6 Issue. 12, December- 2021, pg. 1-17}

ISSN: 2519-9889

Impact Factor: 5.365

Due to the advantages presented, oral delivery systems are still the most preferred technique for delivering API. Despite these benefits, oral administration systems have numerous drawbacks, including low drug stability in the gastrointestinal tract and first-pass metabolism. The transdermal route has been investigated as a potential route for improving medication delivery in order to potentially overcome some of these drawbacks[4]. The risk of liver malfunction and gastrointestinal tract irritation as side effects is low since medications delivered via transdermal delivery systems avoid the gastrointestinal tract and hence avoid conversion by the liver. Other advantages of drug administration through the skin include maintaining an effective rate of drug delivery in time, a consistent rate of circulation, and the advantages of a passive delivery mechanism and diffusion [5].

The most difficult part of developing a transdermal drug delivery system is overcoming the stratum corneum's barrier effect, delivering the drug to skin tissue, and passing through cellular and vascular tissue to reach the target region. The issue is that skin tissue can only transport a minimal amount of the medication. Various innovative transdermal drug delivery strategies have been intensively studied to overcome these challenges and have emerged as desirable administration routes. Furthermore, in terms of the administered dose, cost-effectiveness, and therapeutic efficacy, such development could provide a competitive advantage over existing drug administration strategies. [6]

Because of a variety of enticing qualities, nanostructured lipid carriers (NLC) have been promoted as feasible carriers for transdermal medication administration for the past decade. Traditional topical medicines have a variety of drawbacks, including impermeability of the epidermal barrier, limited efficacy, and frequent administration. Part of the researcher's current focus in the pharmaceutical and cosmetic industries is on developing NLC for topical and dermal applications. NLCs are lipids that are physiologically active, biodegradable, low in toxicity, and contain a variety of advantageous qualities. The low therapeutic efficacy and adverse side effects of conventional topical carriers can be solved by using dermal NLC. [7]

Cubosomes are bicontinous cubic liquid crystalline phase particles that are unique, sub-micron nanostructured particles. They are nanoparticles that are self-assembled liquid crystalline particles of specific surfactants that have an appropriate water ratio and have a solid-like rheology. Cubosomes have the same microstructure as the parent cubic phase, but have a greater specific surface area and smaller viscosity dispersions than the bulk cubic phase. Cubosomes are commonly made by dispersing bulk-cubic phase at a high energy, then stabilising the colloidal phase with polymeric surfactants. Lipids, surfactants, and polymer molecules with both polar and non-polar components constitute these vesicles. Amphiphilic molecules in polar compounds spontaneously self-assemble into an array of thermodynamically stable liquid crystalline phases with lengths on the nanometer scale due to the hydrophobic effect. Cubosomes are thus bicontinous cubic liquid phases that include two distinct water regions separated by surfactant-controlled bilayers. Cubic phases are more bioadhesive, making them ideal for topical and mucosal depositions for drug delivery. Topical delivery methods focus on the use of liquid crystal's unique characteristics and Liquid Crystal Nanoparticle Technology (LCNT). This intriguing technology creates a thin surface coating at surfaces made up of a liquid crystal matrix with a nanostructure that can be adjusted for an ideal delivery profile and provides good temporary protection for painful and sensitive skin. [8]

The most effective treatment for psoriasis is topical steroids, which are quite efficient in treating mild to moderate illness. In moderate to severe disease, they can be administered alone or in conjunction with other drugs. Corticosteroids depress the immune system by decreasing lymphatic system function, decreasing 


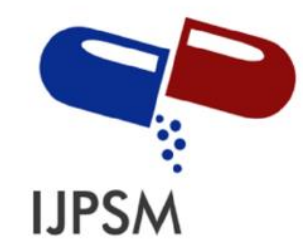

Dr. C.Pandian et al, International Journal of Pharmaceutical Sciences and Medicine (IJPSM), Vol.6 Issue. 12, December- 2021, pg. 1-17

ISSN: 2519-9889

Impact Factor: 5.365

immunoglobulin and complement concentrations, precipitating lymphocytopenia, and interfering with antigenantibody interaction. Triamcinolone is a topical corticosteroid that is used to treat a number of skin problems (e.g., eczema, psoriasis, dermatitis, allergies, rash, and mouth ulcers). It is a low-solubility, low-permeability BCS Class IV medication. When used orally, triamcinolone undergoes substantial first-pass metabolism. Thus from above listed characteristics of drug and taking into account that for psoriasis disease first line of treatment is done by topical route the current study is focused on the development of Cubic nanoparticles for enhanced transdermal delivery. $[9,10]$

\section{MATERIALS REQUIRED}

Triamcinolone was obtained as a gift sample from Pharmafabrikon, Madurai. Poloxamer 407 was obtained from Apex Laboratories Private Limited.,Chennai. HPMC was obtained as a gift sample from Shasun Pharmaceuticals Limited,Chennai. Glyceryl monooleate, Glycerol and ethanol were purchased from Universal scientific appliances, Madurai. All chemicals used were of analytical grade and double distilled water was used throughout the experiments.

\section{METHODOLOGY}

\section{PREPARATION OF CUBOSOMES}

The bottom-up technique was used, in which the nanostructure building blocks were first formed and then gathered into the final substance. It is a more recently established method of cubosome generation that allows cubosomes to synthesize and crystallise from molecular precursors. The sonication approach was chosen for subsequent research because of maximum entrapment efficiency and the smallest particle size is possible.

Glyceryl mono oleate and Poloxamer 407 were used to make the cubosomal formulation. Glyceryl mono oleate was altered from $2.5 \%$ to $5 \%$, while Poloxamer 407 was varied from $0.5 \%$ to $2 \%$, resulting in twelve separate batches. GMO was gently melted in a water bath at $70^{\circ} \mathrm{C}$, then injected dropwise into a preheated poloxamer 407 solution at $70^{\circ} \mathrm{C}$, and drug $5 \mathrm{mg}$ was added before gradually adding distilled water to make up to $20 \mathrm{~mL}$. For 5 minutes, these solutions were mechanically stirred at $1500 \mathrm{rpm}$. After cooling to ambient temperature, the dispersions were sonicated for 5 minutes at maximum power of $120 \mathrm{~W}$. Cubosome milky white dispersions were formed after a 24-hour equilibration period. [11]

\section{EVALUATION OF CUBOSOMES}

\section{Particle size and Polydispersity index}

The particle size and polydispersity index of cubosomes were determined using a computerised zeta sizer device (Malvern Mastersizer 2000 Ver 2.00) at $25^{\circ} \mathrm{C}$ using the dynamic light scattering approach. The zeta sizer cell was filled with cubosomal formulation and the size and Polydiepersity index were measured. [12]

\section{Zeta Potential}

The levels of electrophoretic mobility (zeta potential) of cubosomal dispersions were determined using a zeta sizer and the values were obtained. At $25{ }^{\circ} \mathrm{C}$, the zeta potential was measured using Zetasizer. The samples were stored in a polystyrene cuvette, and the zeta potential was measured using a zeta dip cell. Zeta potential for a stable formuluation is found to be $+/-30 \mathrm{mV}$. 


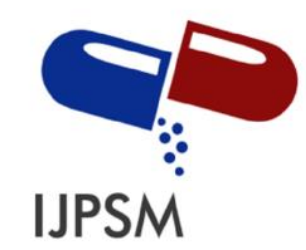

Dr. C.Pandian et al, International Journal of Pharmaceutical Sciences and Medicine (IJPSM), Vol.6 Issue. 12, December- 2021, pg. 1-17

ISSN: 2519-9889

Impact Factor: 5.365

\section{Entrapment efficiency}

The centrifugation method was used to determine the entrapment effectiveness of the developed cubosomal mixture. Cubosomes were centrifuged for 1 hour at $10000 \mathrm{rpm}$ at room temperature. Supernatant containing unentrapped medication in cubosomes was isolated and analyzed against phosphate buffer using a UV spectrophotometer at $\lambda_{\max } 239 \mathrm{~nm}$ (PH 7.4). After rupturing the cubosomes, the remaining entrapped drug was quantified using Triton X 100. [13]

The amount of drug entrapped in cubosomes was determined by calculating the entrapment efficiency by the equation:

$\%$ Entrapment efficiency $=$ Total drug content - Drug content in supernatant X 100

Total drug content

\section{Drug Content}

The drug content of cubosomal formulation was determined by mixing the formulation with methanol and sonicated for 10 minutes to obtain a clear solution and filtered. The filtrate was analysed for drug content by $\mathrm{UV}$ at $\lambda \max 239 \mathrm{~nm}$.

$$
\text { Drug content }=\frac{\text { Actual yield } \times 100}{\text { Theoretical yield }}
$$

\section{Scanning Electron Microscopy}

A scanning electron microscope was used to examine the surface features of prepared cubosomes for morphology. This image was used to confirm the cuboidal geometry of the formulations.10 $\mu$ of cubosomes were uniformly dispersed on a glass slide and allowed to dry at room temperature for SEM visualisation. The morphology was studied with a Philips 505 electron microscope at an accelerating voltage of $2.0 \mathrm{kV}$ after gold coating the sample with a Polaron E5100 gold sputter coater. [14]

\section{In-vitro release study}

The dialysis membrane was used in the release study. The cubosomal formulation was placed on the dialysis membrane and the ends were clamped shut before being suspended in phosphate buffer ph 7.4 in a beaker.The samples were taken at predetermined intervals $(1,2,3,4,5,6,7,8,9,10,11,12$, and 24 hours) and the same volume of buffer solution was immediately replaced to keep the sink condition. Using a UV Spectrometer, the materials were examined at $\lambda_{\max } 239 \mathrm{~nm}$. The data were plotted on a graph with time on the $\mathrm{X}$-axis and percent cumulative medication release on the Y-axis. [13]

\section{In-vitro Permeation study}

Vertical Franz diffusion cells were used to determine In-vitro Permeabilty of the selected best formulation of Cubosomes using the goat skin obtained from the local slaughter house. The skin was stored in a freshly prepared saline solution for 24 hours a day before the study. An epidermal layer of surface area of $1.5 \mathrm{~cm}^{2}$ from the goat skin was placed facing the donor compartment. About $10 \mathrm{ml}$ of Cubosomal formulation was placed on the donor compartment and the receptor compartment was filled with $100 \mathrm{ml}$ of buffer $\mathrm{pH} 7.4$ maintained at $37 \pm 1^{\circ} \mathrm{C}$. Then this design was placed on the magnetic stirrer and maintained at $100 \mathrm{rpm}$. Samples 


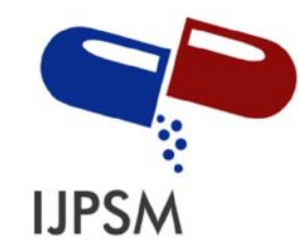

Dr. C.Pandian et al, International Journal of Pharmaceutical Sciences and Medicine (IJPSM), Vol.6 Issue. 12, December- 2021, pg. 1-17

ISSN: 2519-9889

Impact Factor: 5.365

were withdrawn at pre-determined inetervals (at 1,2,3,4,5,6,7,8,9,10,11,12 and $24 \mathrm{~h}$ ) from the receptor compartment and replaced immediately with equal volumes of fresh buffer solution. The samples obtained were analyzed using UV Spectrometer at $239 \mathrm{~nm}$ as $\lambda_{\max }$. The cumulative percentage of drug permeated per unit area $(\mu \mathrm{g} / \mathrm{cm} 2)$ of the skin was plotted as a function of time (h) and the slope was calculated from the linear portion of the curve. The flux $(\mu \mathrm{g} / \mathrm{cm} 2)$ at steady state was calculated by dividing the slope by area of the skin surface through which permeation took place.[15]

\section{Kinetic study}

The first order mode, zero order model, Higuchi, and Korsemeyer-Peppas kinetic models were used to fit the release data of the selected formuation. $\mathrm{n}=0.5$ for fickian diffusion, for anomalous transport $\mathrm{n}=0.5-1$ and for case II transport, $\mathrm{n}=1 .[12]$

\section{FORMULATION OF CUBOSOMES LOADED TRANSDERMAL PATCH}

The solvent evaporation process was used to develop the transdermal patch. iThe hydroxy propyl methyl cellulose was dissolved in ethanol and magnetically stirred until it reached a semisolid consistency. The drug was mixed in these solvents as a cubosomal dispersion and agitated constantly. As a plasticizer, glycerol was added drop by drop to this composition. Poured it onto petridish, covered it with the inverted funnel, and let it dry for 24 hours to obtain a patch. After 24 hours, the patches were removed using a sharp knife inserted along the edge of the patch and stored for future research. [16]

\section{EVALUATION OF TRANSDERMAL PATCHES}

\section{Physical appearance}

Prepared patches were visually inspected for colour, clarity, flexibility and smoothness.[17]

\section{Thickness uniformity}

Thickness of the patch was determined by using digital thickness gauge. The thickness was measured at 4 different points and the average thickness was obtained. [18]

\section{Folding Endurance}

For the prepared patches, the folding durability was carefully measured. A $1 \mathrm{~cm} 2$ patch strip was cut and folded repeatedly in the same spot until it snapped. The value of folding endurance is determined by the number of times the patch may be folded in the same location without breaking or cracking. [17]

\section{Percentage Moisture Absorption}

The patch were weighed accurately and placed in dessicators for 72 hours and after that the patches were reweighed and the percentage moisture absorption was calculated using the formula:

$\%$ Moisture content $=$ Initial weight - Final weight X 100

Initial weight

\section{Drug content}

Short circular patch were cut out into small pieces and soaked in $100 \mathrm{ml}$ of methanol and stirred using magnetic stirrer consecutiovely for 36 hours and sonicated for about 36 minutes. The solution was filtered and evaluated using UV Spectrophotometer at wavelength 239nm.[19] 


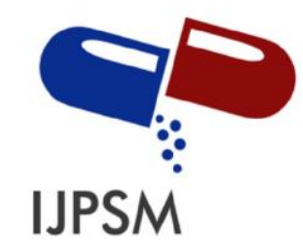

Dr. C.Pandian et al, International Journal of Pharmaceutical Sciences and Medicine (IJPSM), Vol.6 Issue. 12, December- 2021, pg. 1-17

ISSN: 2519-9889

Impact Factor: 5.365

\section{Flatness test}

Each film was divided into longitudinal strips, one in the centre and two on either side. The length of each strip was measured, as well as the variation in length caused by nonuniformity in flatness.

\section{In-vitro release study}

The drug release from the formulated patches were evaluated using USP (apparatus I) Basket type apparatus. The patches were placed in their appropriate baskets, with their drug matrix exposed to phosphate buffer ( $\mathrm{pH} 7.4$ ). All dissolution tests were carried out at $37 \pm 0.5^{\circ} \mathrm{C}$ and $100 \mathrm{rpm}$, using $900 \mathrm{~mL}$ of buffer in each dissolving jar. Samples were taken at various intervals $(1,2,3,4,5,6,7,8,9,10,11,12$, and 24 hours) and evaluated against a blank using a UV Spectrophotometer at $239 \mathrm{~nm}$. For the formulations, cumulative amounts of drug released were plotted against time. [20]

\section{In-vitro Permeation study}

Vertical Franz diffusion cells were used to determine In-vitro Permeabilty of cubosomes loaded transdermal Patches using the goat skin obtained from the local slaughter house. The skin was stored in a freshly prepared saline solution for 24 hours a day before the study. An epidermal layer of surface area of $1.5 \mathrm{~cm}^{2}$ from the goat skin was placed facing the donor compartment. A section of cubosomal patch was placed on the donor compartment and the receptor compartment was filled with $100 \mathrm{ml}$ of buffer $\mathrm{pH} 7.4$ maintained at $37 \pm 1^{\circ} \mathrm{C}$. Then this design was placed on the magnetic stirrer and maintained at $100 \mathrm{rpm}$. Samples were withdrawn at predetermined inetervals (at 1,2,3,4,5,6,7,8,9,10,11,12 and $24 \mathrm{~h}$ ) from the receptor compartment and replaced immediately with equal volumes of fresh buffer solution. The samples obtained were analyzed using UV Spectrometer at $239 \mathrm{~nm}$ as $\lambda_{\max }$. The cumulative percentage of drug permeated per unit area $(\mu \mathrm{g} / \mathrm{cm} 2)$ of the skin was plotted as a function of time (h) and the slope was calculated from the linear portion of the curve. The flux $(\mu \mathrm{g} / \mathrm{cm} 2)$ at steady state was calculated by dividing the slope by area of the skin surface through which permeation took place.[15]

\section{Kinetic study}

The first order mode, zero order model, Higuchi, and Korsemeyer-Peppas kinetic models were used to fit the release data of the selected formulation. $\mathrm{n}=0.5$ for fickian diffusion, for anomalous transport $\mathrm{n}=0.5-1$ and for case II transport, $\mathrm{n}=1 .[12]$

\section{RESULTS AND DISCUSSION PREPARATION OF CUBOSOMES}

The effect of GMO concentration on physical appearance and stability of cubosomal nanoparticles were studied by adding various concentration of GMO such as $2.5 \%, 3.75 \%, 5 \% .10 \%, 15 \%$ and $20 \%$.From the results $2.5 \%, 3.75 \%, 5 \% \mathrm{v} / \mathrm{v}$ of GMO was suitable for cubosomal nanoparticles due to desired physical appearance and stability. Various concentrations $(0.5 \%, 1 \%, 1.5 \%$ and $2 \%)$ of stabilizer were optimised and results showed that $0.5 \%, 1 \%, 1.5 \%$ was sufficient to obtain a stable cubosome formulation. 


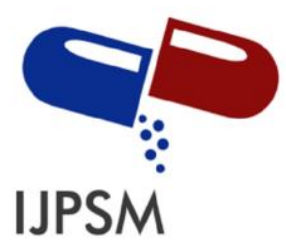

Dr. C.Pandian et al, International Journal of Pharmaceutical Sciences and Medicine (IJPSM), Vol.6 Issue. 12, December- 2021, pg. 1-17

ISSN: 2519-9889

Impact Factor: 5.365

Table 1 : Formulation codes with quantities

\begin{tabular}{|c|c|c|c|c|c|c|c|c|c|c|c|c|}
\hline Formulation code & F1 & F2 & F3 & F4 & F5 & F6 & F7 & F8 & F9 & F10 & F11 & F12 \\
\hline Drug (mg) & 5 & 5 & 5 & 5 & 5 & 5 & 5 & 5 & 5 & 5 & 5 & 5 \\
\hline GMO (g) & 2.5 & 2.5 & 2.5 & 2.5 & 3.75 & 3.75 & 3.75 & 3.75 & 5 & 5 & 5 & 5 \\
\hline Poloxamer 407 (g) & 0.5 & 1 & 1.5 & 2 & 0.5 & 1 & 1.5 & 2 & 0.5 & 1 & 1.5 & 2 \\
\hline
\end{tabular}

\section{EVALUATION OF CUBOSOMES}

\section{Particle size and Polydispersity index}

The particle size range of all formulations are within the nanometer range. The particle size was found to be in the range of $163-357.3 \mathrm{~nm}$ and PDI was found to be in the range of $0.244-0.642$.The results are shown in table 3 and the image of the optimised formulation measurement is shown in figure 3 .

\section{Zeta Potential}

The results obtained here indicates that there is no aggregation in the formulations due to usage of surface stearic stabilizer, poloxamer 407. It produces an envelope around the surface of the nanoparticles and protects from aggregation. Hence the formulation (F6) was observed to be better stable than other formulations. The results are shown in table 3.The image of the zeta potential measurement of the optimised formulation was shown in figure 4.

\section{Entrapment efficacy}

The entrapment efficacy of the formulations increased when higher amounts of GMO was used due to the lipophilic nature of the drug. The enhanced lipid values would improve the solubilisation of the lipophilic drug and provides more space for the entrapment of drug. F6 formulation exhibited maximum entrapment efficiency and the results are shown in table 3.

\section{Drug content}

F6 has the greater content of drug in it $93.66 \pm 3.22$ and it is suitable for further studies. The results are shown in table 3 .

\section{Scanning electron Microscopy}

No aggregation was observed among the particles and the particle surface was smooth without surface deformations and visible pinholes. . The image was shown in figure 2.

\section{In-vitro release studies}

Sustained drug delivery of the drug was provided since it produced a maximum of $39.61 \%$ of release at the end of 24 hours. High entrapment of the lipophilic drug in the lipid matrix attributed to the sustained release. F6 achieved the highest release and it is considered for further studies. The results are shown in table 3 and the graph is shown in figure 5 . 


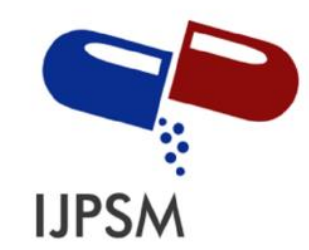

Dr. C.Pandian et al, International Journal of Pharmaceutical Sciences and Medicine (IJPSM), Vol.6 Issue. 12, December- 2021, pg. 1-17

ISSN: 2519-9889

Impact Factor: 5.365

\section{Selection of best formulation}

Optimum particle size, good entrapment efficacy and stability are all desired for good penetration of the formulation across the skin. Considering these factors, formulation F6 was found to be the best formulation, since it has a particle size of $219.3 \mathrm{~nm}$ and $91.5 \%$ entrapment efficacy and was used for further studies.

\section{In-vitro permeation study}

In-vitro permeation study was carried out for the selected best formulation (F6) using goat skin. The Permeation occurs in two steps, initial transfer of drug from the formulation on to the surface of the skin and finally the drug was transferred from the surface of the skin to the receptor containing buffer solution. The amount of drug permeated across the skin was found to be $98.35 \%$. Enhanced Permeability across the skin may be due to the cubic structure of the particles, their small size, lipid content of the cubosomes and presence of surfactants. The results are shown in table 4 and the graph is shown in figure 6.

\section{Kinetic study}

The korsemeyer peppas kinetic plots were found to be fairly linear as indicated by their highest regression values (0.953) for optimised cubosome formulation. As per peppas model 0.953 is the " $n$ value" in order to characterise the different release mechanisms. In this release $\mathrm{n}$ value was found to be nearly 1 for mass transfer following a non fickian model. The results are shown in table 5.

\section{FORMULATION OF CUBOSOMES LOADED TRANSDERMAL PATCH}

The cubosomal patch was prepared using optimised formulation F6 .It was loaded in the patch by calculating the amount of drug needed to be loaded as per the formula. HPMC and ethanol provided good consistency and Glycerol assisted in plasticizer activity. The formulated patch was stored by packing in butter paper and wrapped in an aluminium foil sheet. (Figure 1)

Total amount of drug to be loaded $=$ Area of petridish $\times$ Desired drug

Area of small circular patch

Table 2: Preparation of transdermal patch

\begin{tabular}{|c|c|}
\hline Ingredients & Quantity \\
\hline HPMC & $200 \mathrm{mg}$ \\
\hline Ethanol & Q.S \\
\hline Glycerol & $0.5 \mathrm{ml}$ \\
\hline Drug loaded Cubosomes & $2 \mathrm{ml}$ \\
\hline
\end{tabular}




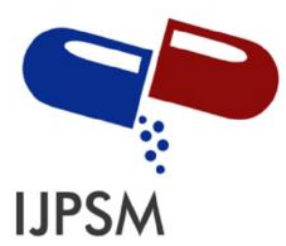

Dr. C.Pandian et al, International Journal of Pharmaceutical Sciences and Medicine (IJPSM), Vol.6 Issue. 12, December- 2021, pg. 1-17

ISSN: 2519-9889

Impact Factor: 5.365

\section{EVALUATION OF TRANSDERMAL PATCHES}

\section{Physical appearance}

White in colour, smooth and flexible.

\section{Thickness uniformity}

A measuring caliper was used to measure the thickness at 3 different areas and from the results it was indicated that the patch has better uniformity in terms of its thickness. It exhibited an average thickness of $1.4 \mathrm{~mm}$.

\section{Folding endurance}

By measuring the formulated patch by folding reveals that it has the ability of 20 times to withstand folding without breaking or visible damage of cracks. The results indicate satisfactory good strength and flexibility.

Folding endurance $=20$

\section{Percentage Moisture absorption}

The percentage moisture content was found to be $4.6 \%$. The lower the moisture content in the patch helps to protect it from microbial contamination and remains stable as it is completely dried.

\section{Drug content}

Drug content was calculated as $96 \%$ in the formulated patch.

\section{Flatness test}

The patches exhibited 0\% constriction and no amount of constriction in the prepared patches indicates $100 \%$ flatness. Thus, these patches could maintain a uniform and smooth surface when they are applied onto the skin.

\section{In-vitro release study}

The drug release from the patch was found to be $40.64 \%$ at the end of 24 hours. It helps in avoiding frequent administration of patches as it exhibited the sustained release. The results are shown in table 6 and the graph is shown in figure 7.

\section{In-vitro Permeation study}

It showed a better permeability of $94 \%$ at the end of 24 hours across the skin. As Triamcinolone belongs to BCS Class IV with low permeability the formulated cubosomal patch has improved its permeability characteristics. Results were plotted in the graph by taking time in $\mathrm{x}$-axis and \% cumulative drug release in $\mathrm{y}$ axis. The results are shown in table 6 and the graph is shown in figure 8 .

\section{Kinetic study}

The Higuchi kinetic plots were found to be fairly linear as indicated by their highest regression values (0.951) for patch. This value indicates that the release was based on diffusion mechanism. The results are shown in table 7. 


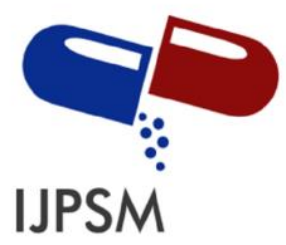

Dr. C.Pandian et al, International Journal of Pharmaceutical Sciences and Medicine (IJPSM), Vol.6 Issue. 12, December- 2021, pg. 1-17

ISSN: 2519-9889

Impact Factor: 5.365

\section{CONCLUSION}

The aforesaid findings demonstrated that cubosomal formulations improved the solubility and permeability of the medication triamcinolone. Because the drug has low solubility and permeability, it was overcome and generated a better result in the form of cubosomes, which boosted the drug's solubility and permeability by a significant amount. The formulation F6 was considered to be the best preparation in terms of Particle size, Polydispersity index, entrapment efficacy, drug content, zeta potential and in-vitro release study and hence it was used in the formulation of transdermal patches. Patches made with the optimised composition produced similar outcomes to cubosomes and were more successful than cubosomal dispersion. As a result, it is concluded that when triamcinolone is administered as a cubosomal patch, its efficacy in the treatment of psoriasis is increased. In the future, more in vivo investigations could be conducted to have a deeper knowledge of the formulations and their properties.

Table 3: Evaluation parameters of Cubosomes

\begin{tabular}{|c|c|c|c|c|c|c|}
\hline Formulation code & $\begin{array}{l}\text { Particle } \\
\text { size }(\mathrm{nm})\end{array}$ & PDI & $\begin{array}{c}\text { Zeta potential } \\
(\mathrm{mV})\end{array}$ & $\begin{array}{c}\text { Entrapment efficacy } \\
(\%)\end{array}$ & $\begin{array}{c}\text { Drug content } \\
(\%)\end{array}$ & $\begin{array}{c}\text { In-vitro release } \\
(\%)\end{array}$ \\
\hline F1 & 275.6 & 0.482 & -28.3 & $86.5 \pm 0.51$ & $88.00 \pm 1.40$ & $26.41 \pm 0.21$ \\
\hline F2 & 225.1 & 0.390 & -29.6 & $88.5 \pm 0.42$ & $87.78 \pm 2.15$ & $21.92 \pm 0.52$ \\
\hline F3 & 221.6 & 0.312 & -36.3 & $83.6 \pm 0.34$ & $89.11 \pm 2.34$ & $23.67 \pm 0.37$ \\
\hline F4 & 357.3 & 0.600 & -33.2 & $82.5 \pm 0.45$ & $87.02 \pm 1.45$ & $23.64 \pm 0.35$ \\
\hline F5 & 327.3 & 0.322 & -34.2 & $90.8 \pm 0.23$ & $88.65 \pm 4.01$ & $34.74 \pm 0.44$ \\
\hline F6 & 219.3 & 0.366 & -43.6 & $91.5 \pm 0.20$ & $93.66 \pm 3.22$ & $39.61 \pm 0.26$ \\
\hline F7 & 217.3 & 0.642 & -34.9 & $88.7 \pm 0.56$ & $89.92 \pm 2.24$ & $34.18 \pm 0.36$ \\
\hline F8 & 280.2 & 0.244 & -22.5 & $89.3 \pm 0.61$ & $89.71 \pm 2.88$ & $30.50 \pm 0.59$ \\
\hline F9 & 190.6 & 0.446 & -27.4 & $91.3 \pm 0.55$ & $87.01 \pm 2.67$ & $37.23 \pm 0.20$ \\
\hline F10 & 165.5 & 0.288 & -38.9 & $90.7 \pm 0.45$ & $89.01 \pm 3.24$ & $27.51 \pm 0.25$ \\
\hline F11 & 163.0 & 0.362 & -36.3 & $90.5 \pm 0.62$ & $86.05 \pm 1.48$ & $19.46 \pm 0.64$ \\
\hline F12 & 226.7 & 0.532 & -16.6 & $91.2 \pm 0.39$ & $86.98 \pm 2.21$ & $19.88 \pm 0.54$ \\
\hline
\end{tabular}

Table 4 : In-vitro release study of the best selected formulation (F6)

\begin{tabular}{|c|c|}
\hline Time (hours) & \% Drug Permeation \\
\hline 1 & $19.87 \pm 0.21$ \\
\hline 2 & $23.68 \pm 0.28$ \\
\hline 3 & $31.90 \pm 0.25$ \\
\hline 4 & $39.66 \pm 0.37$ \\
\hline 5 & $47.98 \pm 0.35$ \\
\hline 6 & $52.42 \pm 0.26$ \\
\hline 7 & $59.02 \pm 0.41$ \\
\hline 8 & $64.73 \pm 0.38$ \\
\hline 9 & $76.26 \pm 0.40$ \\
\hline 10 & $81.63 \pm 0.57$ \\
\hline 11 & $86.81 \pm 0.44$ \\
\hline 12 & $92.41 \pm 0.32$ \\
\hline 24 & $98.35 \pm 0.54$ \\
\hline
\end{tabular}




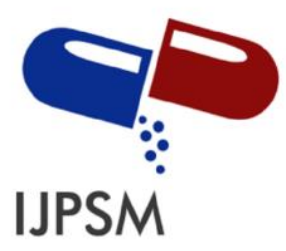

Dr. C.Pandian et al, International Journal of Pharmaceutical Sciences and Medicine (IJPSM), Vol.6 Issue. 12, December- 2021, pg. 1-17

ISSN: 2519-9889

Impact Factor: 5.365

Table 5: Kinetic study of cubosomes (F6)

\begin{tabular}{|c|c|c|c|c|}
\hline \multirow{2}{*}{ Formulation } & \multicolumn{3}{|c|}{ Kinetic Studies } \\
\cline { 2 - 5 } F6 & Zero order & First order & Higuchi model & Korsmeyer Peppas model \\
\hline $\mathrm{R}^{2}$ value & & & & 0.908 \\
& 0.908 & 0.808 & 0.953 & \\
\hline
\end{tabular}

Table 6 : Evaluation parameters of Cubosomes loaded transdermal patch

\begin{tabular}{|c|c|c|}
\hline Time (hours) & In - vitro release (\%) & In-vitro Permeation (\%) \\
\hline $\mathbf{1}$ & 3.69 & 10.60 \\
\hline $\mathbf{2}$ & 6.12 & 14.79 \\
\hline $\mathbf{3}$ & 9.11 & 16.52 \\
\hline $\mathbf{4}$ & 11.16 & 20.47 \\
\hline $\mathbf{5}$ & 13.72 & 31.00 \\
\hline $\mathbf{6}$ & 15.65 & 40.55 \\
\hline $\mathbf{7}$ & 16.99 & 48.10 \\
\hline $\boldsymbol{8}$ & 18.95 & 54.46 \\
\hline $\mathbf{9}$ & 21.18 & 59.89 \\
\hline $\mathbf{1 0}$ & 23.39 & 68.24 \\
\hline $\mathbf{1 1}$ & 29.77 & 72.75 \\
\hline $\mathbf{1 2}$ & 33.08 & 82.45 \\
\hline $\mathbf{2 4}$ & 40.64 & 94.16 \\
\hline
\end{tabular}

Table 7: Kinetic study of Cubosomes loaded transdermal patch

\begin{tabular}{|l|c|c|c|c|}
\hline \multirow{2}{*}{$\begin{array}{c}\text { Transdermal } \\
\text { Patch }\end{array}$} & \multicolumn{3}{|c|}{ Kinetic Studies } \\
\cline { 2 - 5 } & Zero order & First order & Higuchi model & Korsmeyer Peppas model \\
\hline $\mathrm{R}^{2}$ value & 0.951 & 0.934 & 0.951 & 0.934 \\
\hline
\end{tabular}




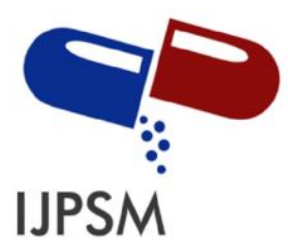

Dr. C.Pandian et al, International Journal of Pharmaceutical Sciences and Medicine (IJPSM), Vol.6 Issue. 12, December- 2021, pg. 1-17

ISSN: 2519-9889

Impact Factor: 5.365

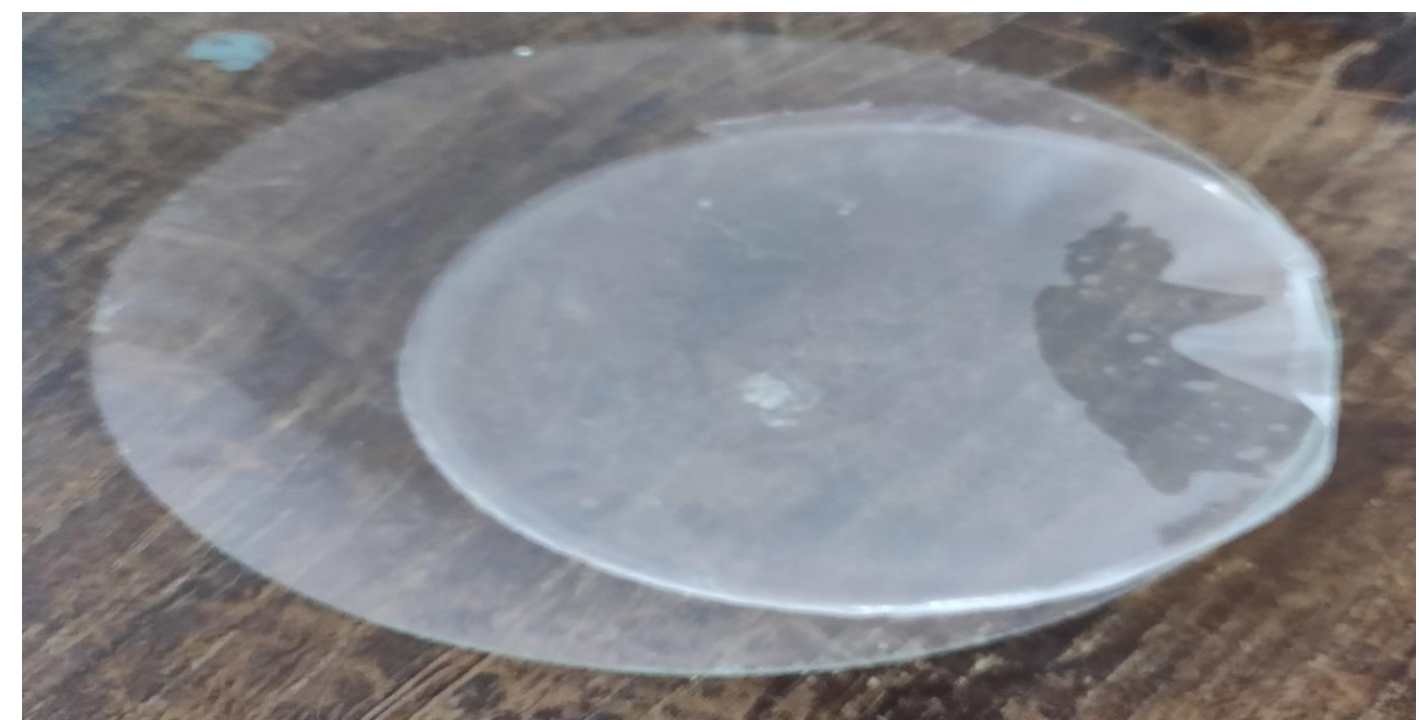

Figure 1: Cubosomes loaded transdermal patch

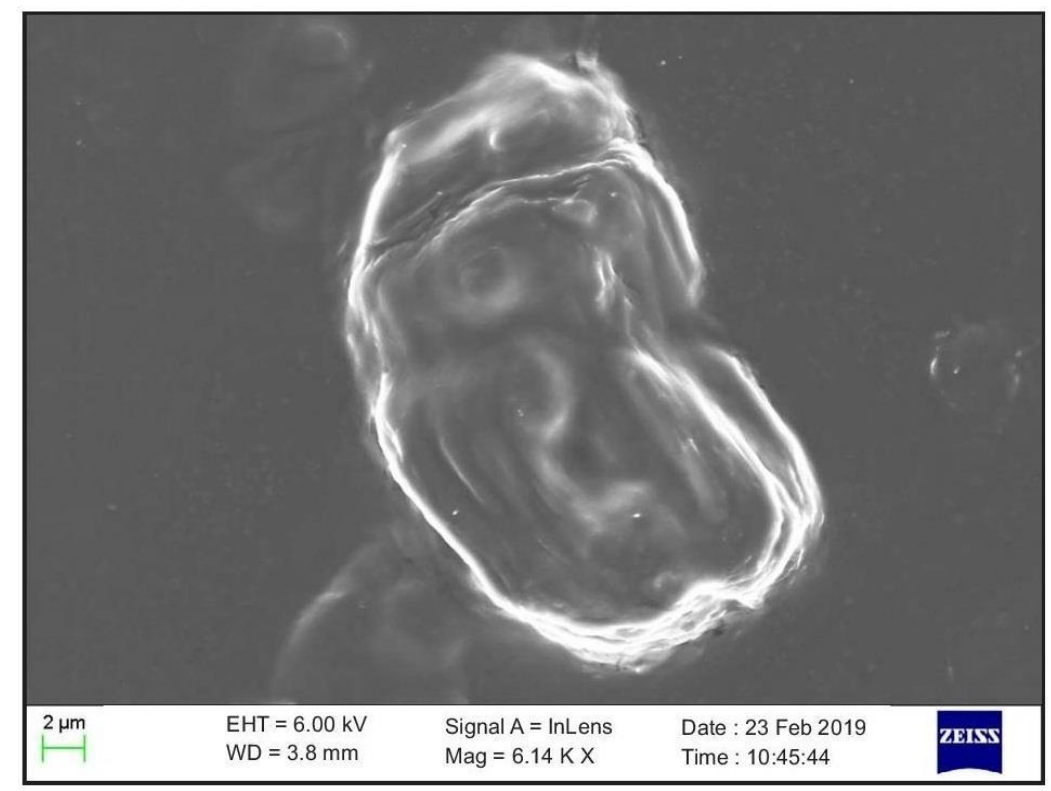

Figure 2: SEM study of Cubosomes 


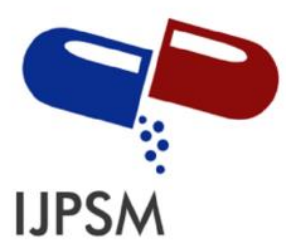

Dr. C.Pandian et al, International Journal of Pharmaceutical Sciences and Medicine (IJPSM), Vol. 6 Issue. 12, December- 2021, pg. 1-17

ISSN: 2519-9889

Impact Factor: 5.365

\section{Size Distribution Report by Intensity}

v2.2

\section{Malvern}

\section{Sample Details}

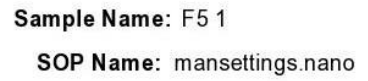

File Name: F3 dts Record Number: 7309

Material RI: 1.59

Material Absorbtion: 0.010
Dispersant Name: Water

Dispersant RI: 1.330

Viscosity (cP): 0.8872

Measurement Date and Time: Monday, February 25, 2019 2:...

System

Temperature $\left({ }^{\circ} \mathrm{C}\right): 25.0$

Count Rate (kcps): 332.6

Duration Used (s): 40

Measurement Position (mm): 4.65

Cell Description: Disposable sizing cuvette

Attenuator: 9

\section{Results}

$\begin{array}{rllll} & & \text { Size (d.nm): } & \text { \% Intensity: } & \text { St Dev (d.n... } \\ \text { Z-Average (d.nm): } 219.3 & \text { Peak 1: } & 277.5 & 88.1 & 123.4 \\ \text { Pdl: } 0.366 & \text { Peak 2: } & 4420 & 6.2 & 914.3 \\ \text { Intercept: } 0.894 & \text { Peak 3: } & 46.13 & 5.8 & 8.472\end{array}$

Result quality : Good

Size Distribution by Intensity

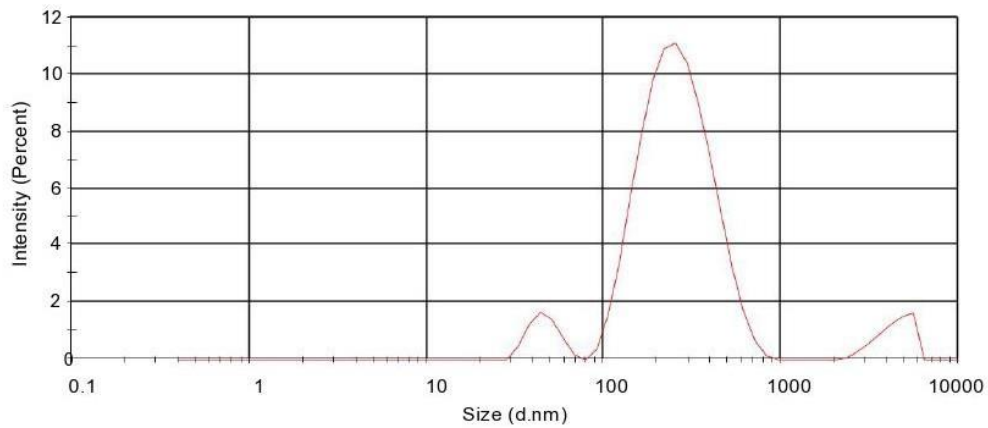

Record 7309: F5 1

Figure 3: Particle size distribution of Cubosomes 


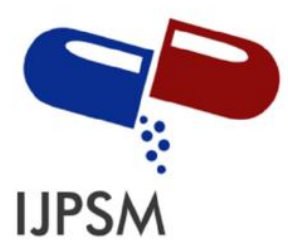

Dr. C.Pandian et al, International Journal of Pharmaceutical Sciences and Medicine (IJPSM), Vol.6 Issue. 12, December- 2021, pg. 1-17

ISSN: 2519-9889

Impact Factor: 5.365

\section{Zeta Potential Report}

v2.3

Malvern Instruments Ltd - $\odot$ Copyright 2008
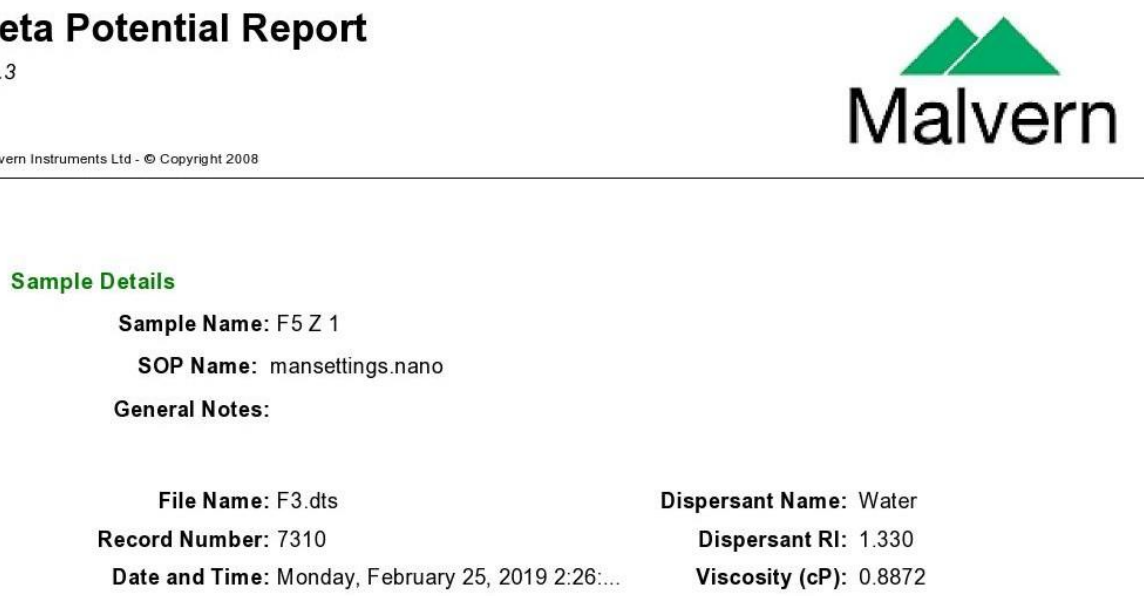

Dispersant Dielectric Constant: 78.5

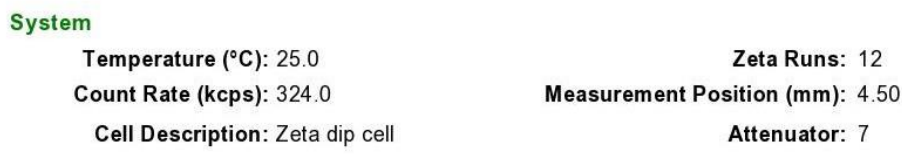

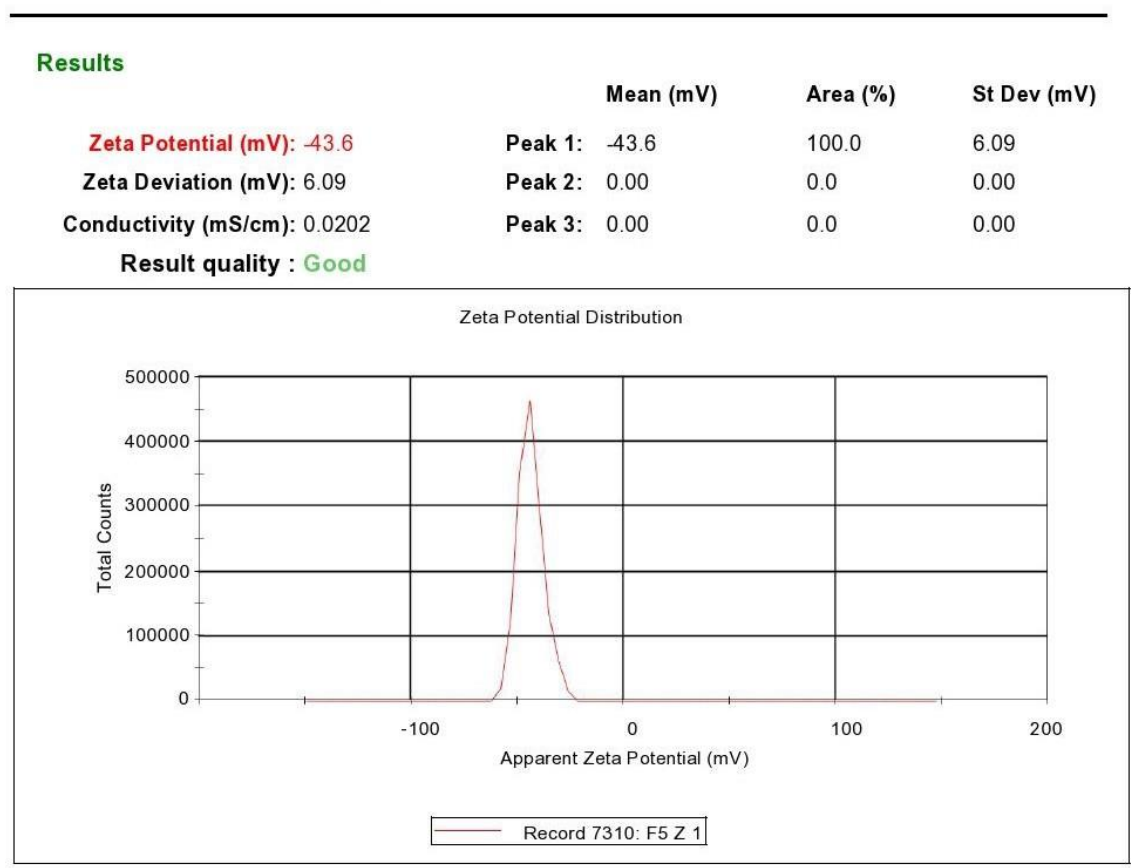

Figure 4: Zeta Potential of Cubosomes 


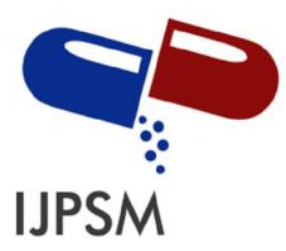

Dr. C.Pandian et al, International Journal of Pharmaceutical Sciences and Medicine (IJPSM), Vol.6 Issue. 12, December- 2021, pg. 1-17

ISSN: 2519-9889

Impact Factor: 5.365

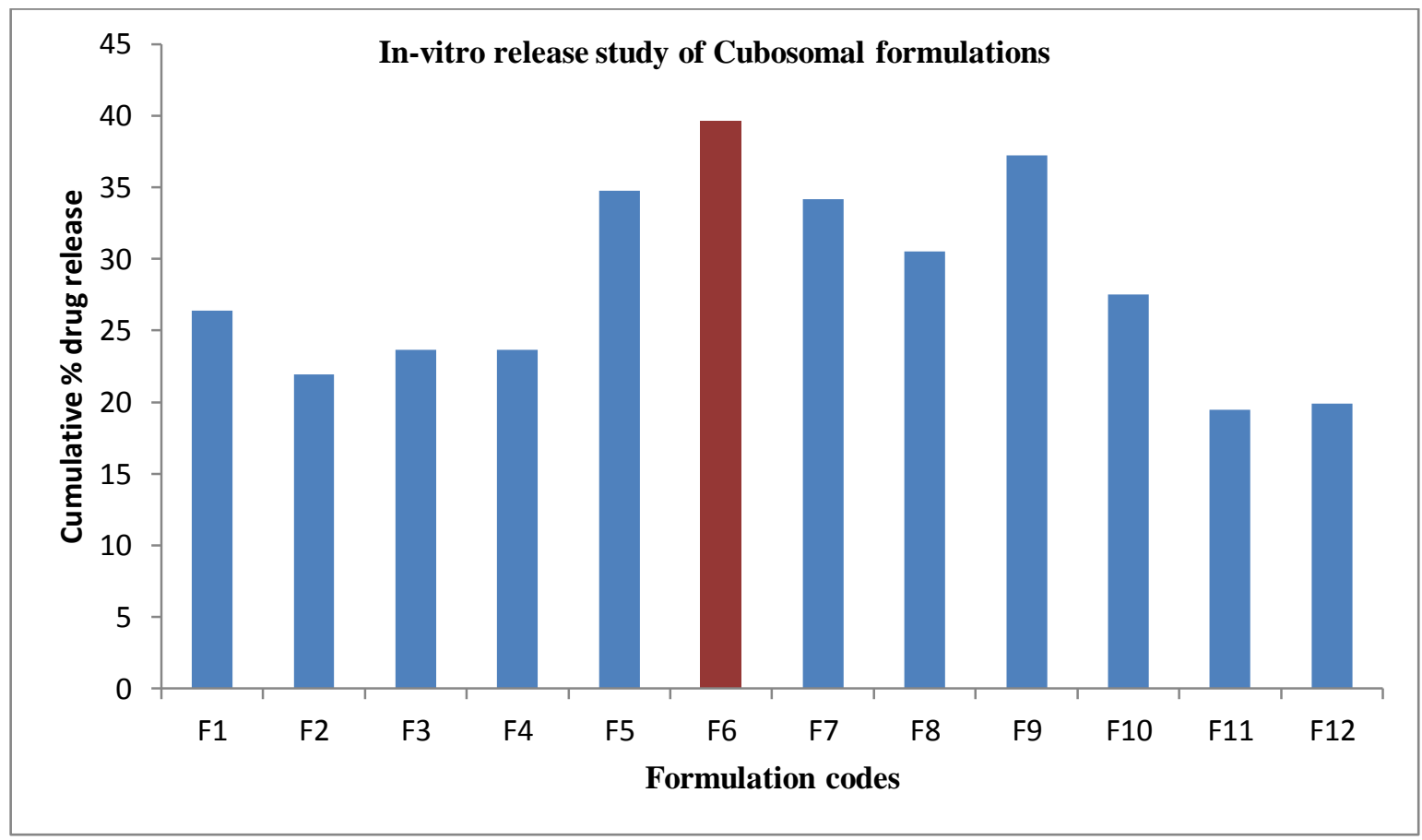

Figure 5: In-vitro drug release of Cubosomes

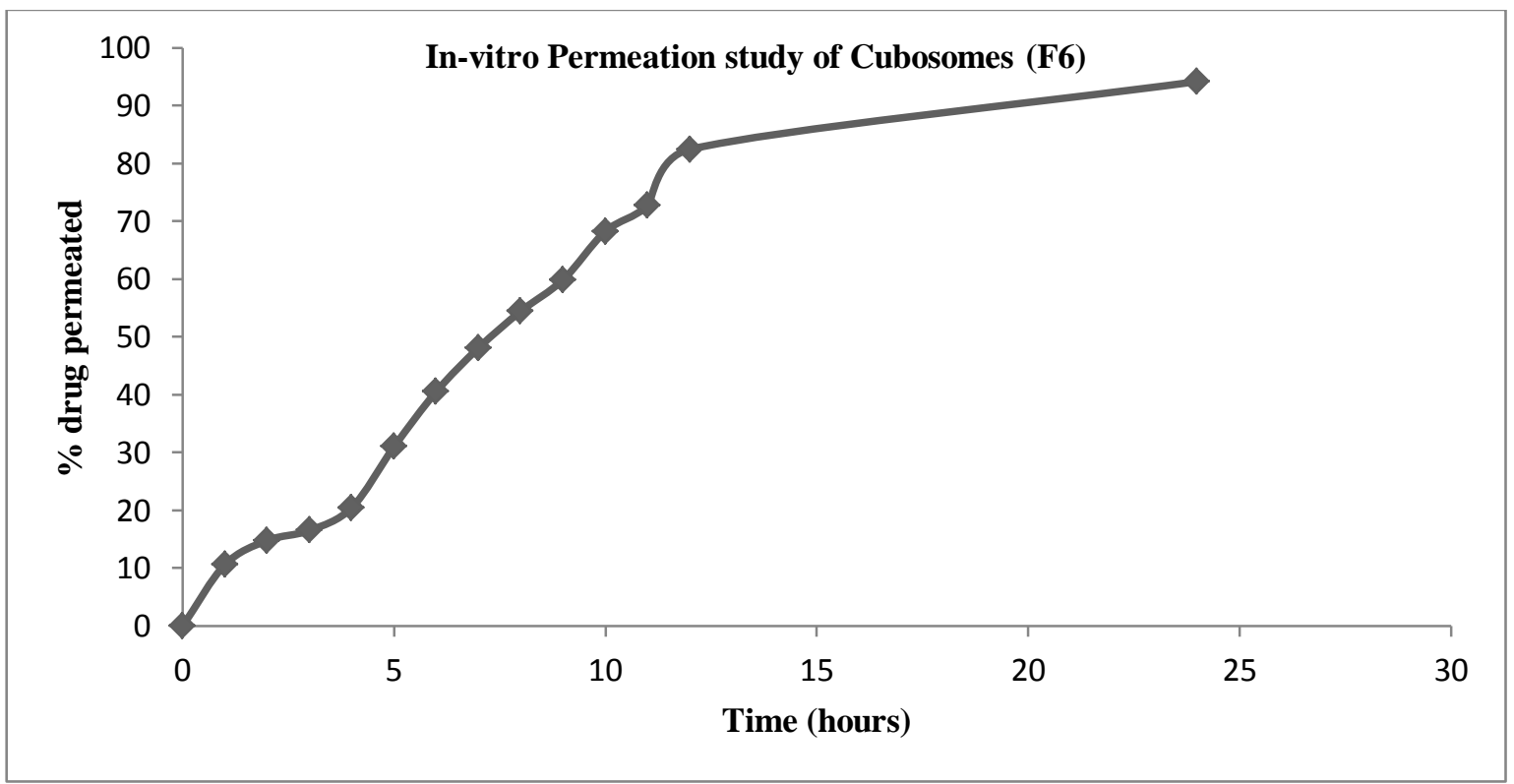

Figure 6: In-vitro permeation of the best selected Cubosomal formulation (F6) 


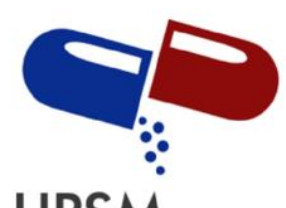

\section{IJPSM}

Dr. C.Pandian et al, International Journal of Pharmaceutical Sciences and Medicine (IJPSM), Vol.6 Issue. 12, December- 2021, pg. 1-17

ISSN: 2519-9889

Impact Factor: 5.365

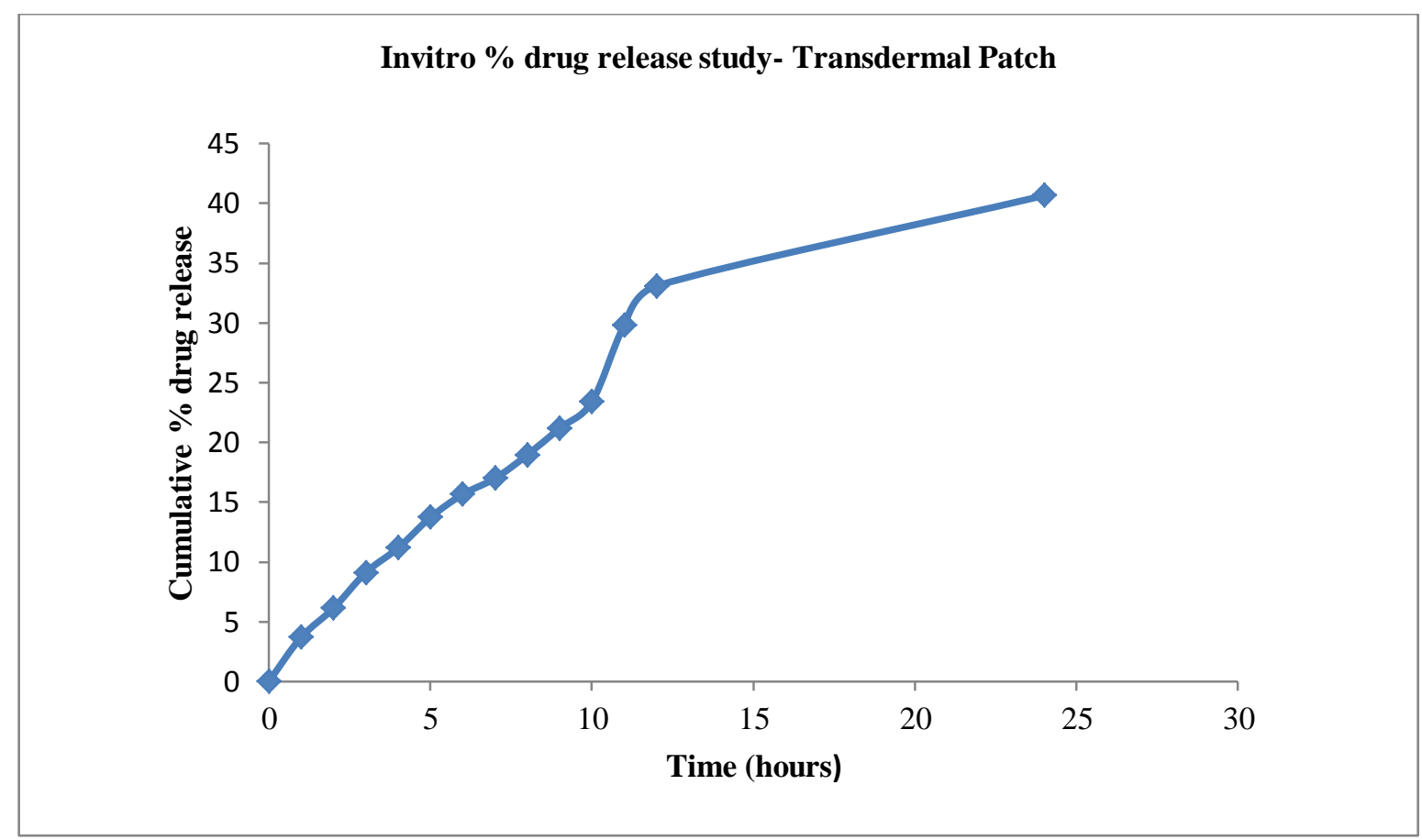

Figure 7: In-vitro release study of Transdermal patch

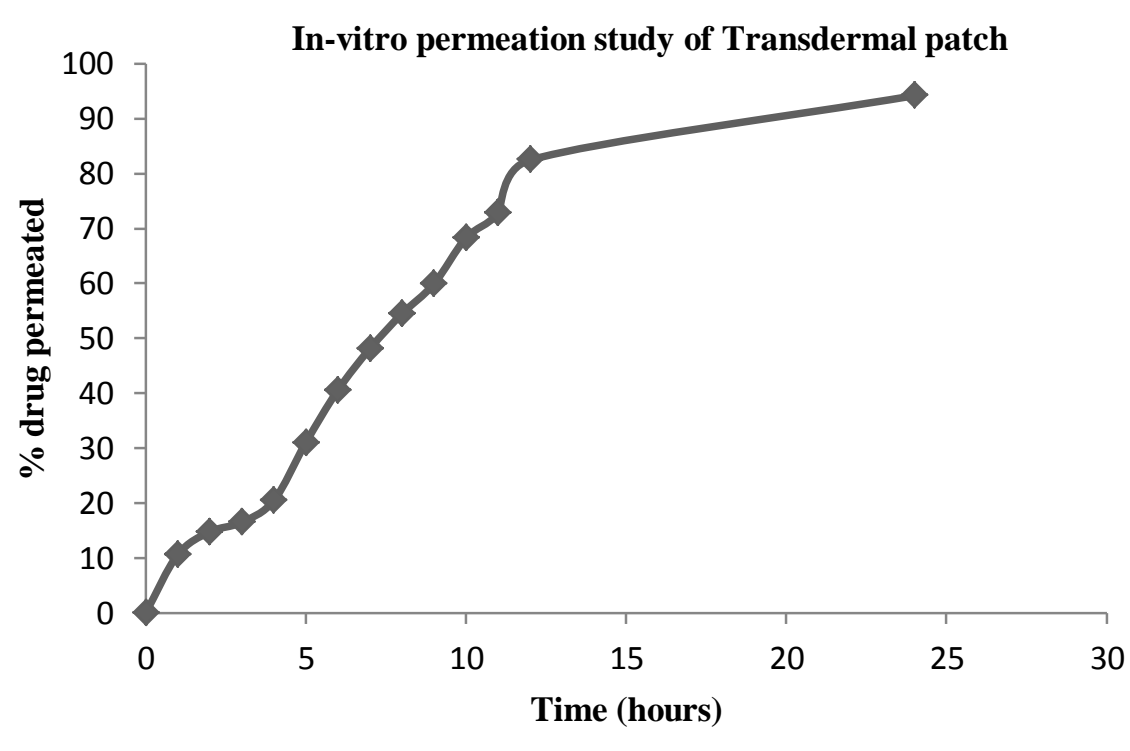

Figure 8: In-vitro permeation of Transdermal patch 


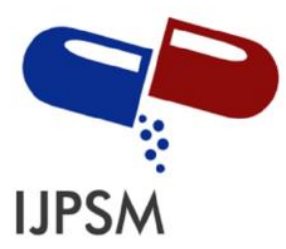

Dr. C.Pandian et al, International Journal of Pharmaceutical Sciences and Medicine (IJPSM), Vol.6 Issue. 12, December- 2021, pg. 1-17

ISSN: 2519-9889

Impact Factor: 5.365

\section{REFERENCES}

[1]. Pragy A. Nair, Talel Badri; Psoriasis [updated 2021 August 11]. In: Statpearls [Internet]. Treasure Island (FL): Statpearls publishing; 2021 Jan.

[2]. Prof. Christopher EM Griffiths, Prof. April W Armstrong, Johann E Gudjonson, Prof. Jonathan NWN Barker; Psoriasis, The Lancet, Vol 397, Issue 10281, P 1301-1315, Apr 2021.

[3]. John Berth Jones; Psoriasis, Medicine; Vol 41, Issue 6, June 2013, Pages 334-340.

[4]. Ryan F. Donnelly, Delly Ramadon, Maeliosa T.C. McCrudden and Aaron J.Courtenay; Enhancement strategies for Transdermal drug delivery system: Current trends and applications; Drug delivery and traditional research, Jan 2021.

[5]. Farzaneh Sabbagh and Beom Soo Kim; Recent advances in polymeric transdermal drug delivery system; Journal of controlled release, Vol 341, Pages 132-146, Jan 2021.

[6]. Ki Su Kim, Hye Eun Chai, Mina Kwon and Woo Yeup Jeong; Recent advances in transdermal drug delivery systems: A review; Biomaterials research; 25:24, 2021.

[7]. Nikam Supriya. S, Phadatre Priya. P, Watode Ankita. B and Kalyani Kayande; Nanostructured lipid carriers: A promising approach for topical drug delivery system; Inetrnational Journal of Pharmaceutical sciences and medicine; Vol 6, Issue 7,Pages 81-101, Jukly 2021.

[8]. Dhadwal Avantika, Sharma Dev Raj, Pandit Vijay, Ashwin Mahendrs singh and Kumar Pravin; Cubosomes : A novel carrier for transdermal drug delivery; Journal of drug delivery and therapeutics; 123-130; 2020.

[9]. Luis Uva, Diana Miguel and Catarina Pinheiro etal., Mechanisms of action of topical corticosteroids in Psoriasis; International Journal of Endocrinology; 2012.

[10].WHO Library Cataloguing- in- publication data; Global report on Psoriasis; World Health Organisation; ISBN $978924156518 ; 2016$.

[11]. Avachat AM, Parpani SS; Formulation and development of bicontinous nanostructured liquid crystalline particles of Efavirenz; Colloids Surf B Biointerfaces; 126: 87-97; Feb 2015.

[12].Hadel Abo El- Enin, Areej Hamed AL- Shanbari; Nanostructured liquid crystalline formulation as a remarkable new drug delivery system of anti-epile[tic drugs for treating children patients; Saudi Pharmaceutical Journal; Vol 26, Issue 6, 790-800; 2018.

[13]. Thoutreddy Rajani, Gullapudi Mahesh, Bonepally Chandra Shekhar Reddy; Formulation and evaluation of Dexamethasone loaded Cubosomes; Research Journal of Pharmacy and Technology; Vol 13, Issue 2, 2020.

[14]. Changquan Calvir Sun, Shuai Qian, Yuan Gao etal., Cubosomes with surface cross-linked chitosan exhibit sustained release and bioavailability enhancement for Vinpocetine; Royal Society of Chemisrty adv., 6287 6298, 2019.

[15].Xinsheng, Yangfang Zhou and Linghui Dianz etal., Characteriztation of Cubosomes as a targeted and sustained transdermal delivery system for Capsaicin; Drug design, development and therapy; 9:4209-4218; 2015.

[16].Lincy John, Arunkumar and Sandra Samuel; Formulation and evaluation of Amlodipine transdermal patches using ethyl cellulose; International research Journal of Pharmacy; 4(10); 2013.

[17].K. Sravanthi, D. Rama Brahma Reddy and A. Srisha etal., Preparation and in-vitro evaluation of transdermal patch of Aceclofenac; American journal of Pharmtech research; 10(02); 2020.

[18]. Dharmesh Trivedi and Anju Goyal; Formulation and evaluation of transdermal patches containing Dexketoprofen trometamol; International journal of pharmaceutical chemistry and analysis; 7(2):87-97; 2020.

[19].Samiullah, Syed Umer Jan, Rahman Gul, Syed Jalaludina and Asmathullah; Formulation and evaluation of transdermal patches of Pseudoephedrine hydrochloride; International journal of applied pharmaceutics; Vol 12, Issue 3, 2020.

[20]. Sahoo Sunit Kumar, Burahari Behury and Patil Sachin kumar; Formulation and evaluation of transdermal patch of Stavudine; Dhaka University journal of Pharmaceutical sciences; 12(1): 63-69; June 2013. 